\title{
Autoimmunity, phospholipid-reacting antibodies and malaria immunity
}

\author{
LR Gomes $^{1, *}$, YC Martins ${ }^{1,2, *}$, MF Ferreira-da-Cruz ${ }^{1}$ and CT Daniel-Ribeiro ${ }^{1}$ \\ ${ }^{1}$ Laboratory for Malaria Research, Instituto Oswaldo Cruz, Fundação Oswaldo Cruz (Fiocruz), Rio de Janeiro and Center for Malaria Research \\ and Training (CPD-Mal), Fiocruz, Rio de Janeiro / Secretary for Health Surveillance (SVS), Ministry of Health, Brazil; and \\ ${ }^{2}$ Department of Pathology, Albert Einstein College of Medicine, The Bronx, New York, USA
}

\begin{abstract}
Several questions regarding the production and functioning of autoantibodies $(\mathrm{AAb})$ during malaria infection remain open. Here we provide an overview of studies conducted in our laboratory that shed some light on the questions of whether antiphospholipid antibodies (aPL) and other AAb associated with autoimmune diseases (AID) can recognize Plasmodia antigens and exert anti-parasite activity; and whether anti-parasite phospholipid antibodies, produced in response to malaria, can inhibit phospholipid-induced inflammatory responses and protect against the pathogenesis of severe malaria. Our work showed that sera from patients with AID containing AAb against dsDNA, ssDNA, nuclear antigens (ANA), actin, cardiolipin (aCL) and erythrocyte membrane antigens recognize plasmodial antigens and can, similarly to monoclonal AAb of several specificities including phospholipid, inhibit the growth of $P$. falciparum in vitro. However, we did not detect a relationship between the presence of anti-glycosylphosphatidylinositol (GPI) antibodies in the serum and asymptomatic malaria infection, although we did register a relationship between these antibodies and parasitemia levels in infected individuals. Taken together, these results indicate that autoimmune responses mediated by AAb of different specificities, including phospholipid, may have anti-plasmodial activity and protect against malaria, although it is not clear whether anti-parasite phospholipid antibodies can mediate the same effect. The potential effect of anti-parasite phospholipid antibodies in malarious patients that are prone to the development of systemic lupus erythematosus or antiphospholipid syndrome, as well as the (possibly protective?) role of the (pathogenic) aPL on the malaria symptomatology and severity in these individuals, remain open questions. Lupus (2014) 23, 1295-1298.
\end{abstract}

Key words: Antiphospholipid syndrome; systemic lupus erythematosus; anti-DNA antibodies; malaria

\section{Introduction}

Despite the increasing knowledge on immunology during the past decades, several questions regarding autoantibody $(\mathrm{AAb})$ production and function remain open. Some of them concern the relationships between autoimmunity and infectious and parasitic diseases. The concept that AAb production and autoimmunity may follow and complicate infectious and parasitic diseases such as Group A B hemolytical streptococcal infection

*LRG and YCM contributed equally to the work

Correspondence to: Cláudio Tadeu Daniel-Ribeiro, Fundação Oswaldo Cruz Avenida Brasil, 4365 Pavillhão Leônidas Deane Rio de Janeiro, 2104-360 Brazil.

Email: ctdr@uol.com.br and Chagas Disease is now well established. ${ }^{1}$ However, evidence has accumulated to indicate that some parasitic diseases, such as malaria, may protect individuals against the development and severity of autoimmune diseases (AID) such as systemic lupus erythematosus (SLE). ${ }^{2,3}$ In addition, it has been proposed that although AAb produced by individuals infected by Plasmodia do not seem to participate in the pathogenesis of malaria, they could even exert protective effects against the disease. $^{4}$

Here we give an overview of studies conducted in our laboratory to approach two of the questions concerning autoimmunity and malaria: (a) Do AID-associated AAb, including those directed to negatively charged phospholipids (antiphospholipid antibodies (aPL)), recognize antigens present 
1296

on Plasmodia and, could these AAb exert an antiparasite activity? and, (b) Can similar (anti-parasite phospholipid (PL) antibodies (Ab)), produced in response to malaria, inhibit PL-induced inflammatory responses and protect against the pathogenesis of severe malaria?

Malaria remains the most prevalent parasitic disease worldwide. All five species of Plasmodia that naturally infect humans can cause from asymptomatic infection to severe disease, but Plasmodium falciparum infection is by far the most prone to cause (and is the most frequently associated with) lifethreatening complications. Clinical manifestations of severe falciparum malaria vary according to transmission intensity and include hyperparasitemia, hypoglycemia, cerebral malaria, severe anemia and respiratory distress.

Many of the malaria-associated symptoms and complications result from a severe host innate immune response induced by parasite and host molecules containing pathogen-associated molecular patterns (PAMPs) released in the circulation during schizogony. P. falciparum glycosylphosphatidylinositol (GPI) is an abundantly expressed PL that activates leukocytes, triggers the release of proinflammatory cytokines and induces the expression of adhesion molecules via toll-like receptors (TLR) -2 and $-4 .^{5}$

It has been demonstrated that the administration of $P$. falciparum GPI in mice induces malaria-like symptoms, ${ }^{6}$ and that the immunization with nontoxic moieties of GPI that elicits an anti-GPI Ab response can prevent malaria pathology and death caused by experimental $P$. berghei ANKA and $P$. yoelii 17XL infection in mice. ${ }^{9,8}$ In addition, an association between the presence of anti-GPI $A b$, age and prevalence of protected asymptomatic individuals has been shown in areas of high malaria transmission in Kenya and north-west Papua New Guinea. ${ }^{9,10}$ Therefore, although not consensually, it has been considered that anti-GPI Ab could reflect and mediate, at least partially, the anti-disease immunity in malaria, by neutralizing the toxic effect of parasite GPI. ${ }^{7}$

Our group also evaluated the association between anti-GPI Ab response and development of clinical malaria ${ }^{11}$ by comparing the incidence of these antibodies, in supposedly immune asymptomatic carriers of plasmodial infection living in an area of the Brazilian Amazon (Barcelos) with high transmission rates of malaria, with controls with no infection or previous history of malaria from the same area and Angolan (Lubango) patients with symptomatic disease. Our data indicated that the $\mathrm{Ab}$ response against $P$. falciparum GPI was not associated with falciparum asymptomatic infection in individuals chronically exposed to malaria in the Brazilian Amazon. We could not rule out that the high levels of anti-GPI Ab, correlated with degrees of parasitemia, observed in Lubango patients with symptomatic, but non-complicated, malaria could be protecting them from severe disease. However, an alternative and more tempting interpretation is that this $\mathrm{Ab}$ response is being boosted by the increasing quantities of $P$. falciparum GPI released in the circulation as the parasitemia level grows (Figure 1). ${ }^{11}$

Hence, our findings do not support the hypothesis that anti-GPI antibodies mediate clinical (antidisease) immunity against malaria, but are in accordance with those of Boutlis et al. ${ }^{12}$ and Cissoko et al. ${ }^{13}$ These authors also failed to detect a relationship between anti-GPI Ab and asymptomatic infections, but, similarly to us, observed a relationship between these $A b$ and parasitemia levels in infected individuals from Mali and Papua New Guinea.

On the other hand, it is becoming progressively clear that autoimmune responses generated during malaria infection are not harmful, but might present protective properties against the disease. Evidence exists for AAb against erythrocytes, ${ }^{14-16}$ PL, ${ }^{17-20}$ brain proteins, ${ }^{21,22}$ and nuclear antigens. ${ }^{20,23-25}$

For the last decade, our team has been collecting evidence that autoimmunity is involved in the protection against malaria. ${ }^{26}$ Zanini et al. ${ }^{23}$ investigated the presence of $\mathrm{AAb}$ in the sera of malarious patients, the reactivity of $\mathrm{AAb}$ from Brazilian autoimmune patients against plasmodial antigens and the effect of such $\mathrm{AAb}$ on the in vitro growth of $P$. falciparum. The results showed that the frequency of sera from malarious patients reacting with actin and the cardiolipin PL was higher $(p<0.001)$ than in sera from SLE patients and, conversely, that antinuclear antibodies (ANA) were more frequent in SLE than in malaria sera $(p=0.004)$. In addition, AAb (directed against dsDNA and ssDNA, nuclear Ag, actin, cardiolipin and erythrocyte membrane) from patients with autoimmune processes recognized plasmodial antigens in the absence of malaria, (44\% of the SLE and $81 \%$ of the malaria - sera reacted with plasmodial Ag by the immunofluorescent antibody test (IFAT)); and $47.3 \%$ of the SLE patients' (and $87.5 \%$ of the malaria patients') sera inhibited plasmodial growth in vitro. The presence of anti-DNA and ANA was important in such anti-plasmodial activity, but no relationship was found between the $P$. falciparum inhibitory growth of 


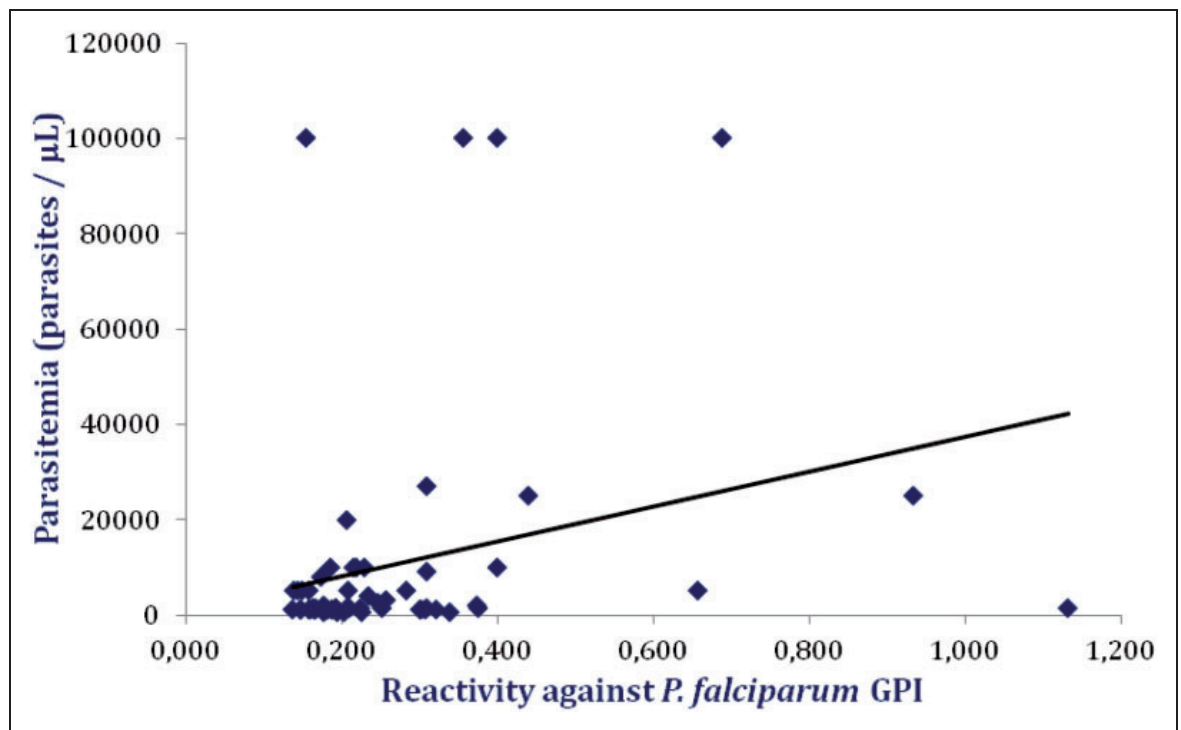

Figure 1 Correlation between the intensity of the immune response directed against the Plasmodium falciparum glycosylphosphatidylinositol (GPI) phospholipid (as assessed by ELISA) and the parasitemia (parasites / microliter) in peripheral blood of acute falciparum malaria patients from Lubango (Angola). Spearman's rank correlation $r=0.3198$ and $p=0.0106$.

immunoglobulins from patients with autoimmune disease and the presence of anti-actin or anti-cardiolipin AAb.

We also studied the relationship between autoimmunity and antimalarial responses by determining - in addition to the presence and the pattern of reactivity to plasmodial antigens of sera from 93 French patients with 14 different AID and not previously exposed to malaria - the effect of 21 monoclonal AAb with different specificities on the in vitro growth to $P$. falciparum $;{ }^{20} 56 \%$ of sera from AID patients and none of healthy individuals reacted with plasmodial antigens. The frequency of sera reacting with at least one of the three strains and two species of Plasmodia depended on the specificity of the $\mathrm{AAb}$ the sera contained, but the differences were not statistically significant. In addition, they reacted more frequently with the young (less differentiated?) rather than with the mature blood stages of Plasmodium. Finally, $85 \%$ of the 13 murine monoclonal AAb reacted with plasmodial antigens and $72 \%$, including three out of four anticardiolipin $\mathrm{AAb}$, inhibited the in vitro growth of $P$. falciparum, although this inhibition did not correlate with the intensity of reactivity against the parasite, as assessed by the IFAT.

Taken together, these results and data from other groups indicate that autoimmune responses may have anti-plasmodial activity, mediated by $\mathrm{AAb}$ of different specificities in the presence or not of other serum factors, and protect against malaria. The potential effect of the theoretically malaria-protective anti-parasite PL in (SLE or anti-PL syndrome-prone) malarious patients as well as the (possibly protective?) role of the (pathogenic) anti-PL AAb in these individuals on the symptomatology and severity of malaria remain open questions.

\section{Funding}

This research received financial support from POM-IOC and PAPES, Fiocruz; CNPq and Faperj, Brazil.

\section{Conflict of interest statement}

The authors have no conflicts of interest to declare.

\section{Acknowledgements}

CTDR and MFFC are recipients of Research Productivity Fellowships from the Conselho Nacional de Desenvolvimento Cientifico e Tecnológico $(\mathrm{CNPq})$ and from the Fundação Carlos Chagas Filho de Amparo à Pesquisa do Estado do Rio de Janeiro (Faperj) as "Cientistas do Nosso Estado". LRG is recipient of a Doctoral Fellowship from the IOC, Fiocruz. YCM is supported by the Inter-hemispheric Research/NIH 
GID (D43 TW007129). The authors are grateful to Professor Roger A. Levy for helpful discussions and technical support on different aspects of the antiphospholipid autoimmune response study and to the Lieutenant Colonel Doctor Mario José Silva de Carvalho from the Instituto de Biologia do Exército (IBEx) for providing plasma for the in vitro culture of $P$. falciparum blood stages and the consequent GPI extraction from cultured parasites.

\section{References}

1 Cunha-Neto E, Teixeira PC, Nogueira LG, Kalil J. Autoimmunity. Adv Parasitol 2011; 76: 129-152.

2 Greenwood BM. Auto immune disease and parasitic infections in Nigerians. Lancet 1968; 1: 380-382.

3 Greenwood B, Herrick EM, Voller A. Suppression of auto-immune disease in NZB and (NZB $\times$ NZW) F1 hybrid mice by infection with malaria. Nature 1970; 226: 266-267.

4 Daniel-Ribeiro CT, Zanini G. Autoimmunity and malaria: What are they doing together? Acta Trop 2000; 76: 205-221.

5 Schofield L, Hackett F. Signal transduction in host cells by a glycosylphosphatidylinositol toxin of malaria parasites. $J$ Exp Med 1993; 177: 145-153.

6 Schofield L, Vivas L, Hackett F, Gerold P, Schwarz RT, Tachado S. Neutralizing monoclonal antibodies to glycosylphosphatidylinositol, the dominant TNF-alpha-inducing toxin of Plasmodium falciparum: Prospects for the immunotherapy of severe malaria. Ann Trop Med Parasitol 1993; 87: 617-626.

7 Schofield L, Hewitt MC, Evans K, Siomos MA, Seeberger PH. Synthetic GPI as a candidate anti-toxic vaccine in a model of malaria. Nature 2002; 418: 785-789.

8 Cherif MS, Shuaibu MN, Kodama Y, et al. Nanoparticle formulation enhanced protective immunity provoked by PYGPI8p-transamidase related protein (PyTAM) DNA vaccine in Plasmodium yoelii malaria model. Vaccine 2014; 32: 1998-2006.

9 Naik RS, Branch OH, Woods AS, et al. Glycosylphosphatidylinositol anchors of Plasmodium falciparum: Molecular characterization and naturally elicited antibody response that may provide immunity to malaria pathogenesis. $J$ Exp Med 2000; 192: 1563-1576.

10 Keenihan SH, Gramzinksi R, Ratiwayanto S, et al. Plasmodium falciparum. Mechanisms of innate and acquired protection against Plasmodium falciparum in Javanese transmigrant adults and children newly resident in malaria-endemic Northwest Papua. Adv Exp Med Biol 2003; 531: 83-102.

11 Gomes LR, Totino PRR, Sanchez MCA, et al. Asymptomatic infection in individuals from the municipality of Barcelos (Brazilian Amazon) is not associated with the anti-Plasmodium falciparum glycosylphosphatidylinositol antibody response. Mem Inst Oswaldo Cruz 2013; 108: 796-800.

12 Boutlis CS, Gowda DC, Naik RS, et al. Antibodies to Plasmodium falciparum glycosylphosphatidylinositols: Inverse association with tolerance of parasitemia in Papua New Guinean children and adults. Infect Immun 2002; 70: 5052-5057.

13 Cissoko Y, Daou M, Lyke KE, et al. Serum antibody levels to glycosylphosphatidylinositols in specimens derived from matched Malian children with severe or uncomplicated Plasmodium falciparum malaria and healthy controls. Am J Trop Med Hyg 2006; 75: 199-204.

14 Schetters TP, Van Run van Breda JH, Hermsen C, Curfs J, Eling WM. Protective and pathological activity in serum of mice developing resistance to Plasmodium berghei infection. Parasite Immunol 1989; 11: 413-423.

15 Hogh B, Petersen E, Crandall I, Gottschau A, Sherman IW. Immune responses to band 3 neoantigens on Plasmodium falciparum-infected erythrocytes in subjects living in an area of intense malaria transmission are associated with low parasite density and high hematocrit value. Infect Immun 1994; 62: 4362-4366.

16 Pantaleo A, Giribaldi G, Mannu F, Arese P, Turrini F. Naturally occurring anti-band 3 antibodies and red blood cell removal under physiological and pathological conditions. Autoimmun Rev 2008; 7: 457-462.

17 Bate CAW, Taverne J, Bootsma HJ. Antibodies against phosphatidylinositol and inositol monophosphate specifically inhibit tumour necrosis factor induction by malaria exoantigens. Immunology 1992; 76: 35-41.

18 Facer CA, Agiostratidou G. High levels of anti-phospholipid antibodies in uncomplicated and severe Plasmodium falciparum and in P. vivax malaria. Clin Exp Immunol 1994; 95: 304-309.

19 Kwiatkowski MD, Bate CAW, Scragg IG, Beattie P, Udalova I, Knight JC. The malarial fever response pathogenesis, polymorphism and prospects for intervention. Ann Trop Med Parasitol 1997; 91: $533-542$.

20 Brahimi K, Martins YC, Zanini GM, Ferreira-da-Cruz M de F, Daniel-Ribeiro CT. Monoclonal auto-antibodies and sera of autoimmune patients react with Plasmodium falciparum and inhibit its in vitro growth. Mem Inst Oswaldo Cruz 2011; 106(Suppl 1): 44-51.

21 Dassé R, Lefranc D, Dubucquoi S, et al. Singular, systemic, selfreactive IgG patterns related to age: Relationship with cerebral malaria susceptibility in exposed subjects residing in an endemic area in Abidjan, Côte-d'Ivoire. Bull Soc Pathol Exot 2012; 105: 276-283.

22 Duarte J, Herbert F, Guiyedi V, et al. High levels of immunoglobulin E autoantibody to 14-3-3 epsilon protein correlate with protection against severe Plasmodium falciparum malaria. J Infect Dis 2012; 206: 1781-1789.

23 Zanini GM, De Moura Carvalho LJ, Brahimi K, et al. Sera of patients with systemic lupus erythematosus react with plasmodial antigens and can inhibit the in vitro growth of Plasmodium falciparum. Autoimmunity 2009; 42: 545-552.

24 Baker VS, Imade GE, Molta NB, et al. Cytokine-associated neutrophil extracellular traps and antinuclear antibodies in Plasmodium falciparum infected children under six years of age. Malar $J$ 2008; 7: 41

25 Mannoor K, Li C, Inafuku M, et al. Induction of ssDNA-binding autoantibody secreting $\mathrm{B}$ cell immunity during murine malaria infection is a critical part of the protective immune responses. Immunobiology 2013; 218: 10-20.

26 Daniel-Ribeiro CT. Is there a role for autoimmunity in immune protection against malaria? Mem Inst Oswaldo Cruz 2000; 95: 199-207. 